\title{
Scavenger Receptor Cysteine-Rich Type 1 Protein M130
}

National Cancer Institute

\section{Source}

National Cancer Institute. Scavenger Receptor Cysteine-Rich Type 1 Protein M130. NCI

Thesaurus. Code C104064.

Scavenger receptor cysteine-rich type 1 protein M130 (1156 aa, 125 kDa) is encoded by the human CD163 gene. This protein plays a role in clearance and endocytosis of hemoglobin/haptoglobin complexes by macrophages. 